\title{
At home with neural stem cells
}

The adult neural stem cells (NSCs) that reside in the walls of the lateral ventricle (LV) are thought to exist in specialized niches that regulate their behaviour. Three recent papers provide new details about the immediate environment and regulation of these NSCs.

Adult NSCs - a subpopulation of the so-called 'type B cells' in the ventricle walls - were thought to be located in the subventricular zone (SVZ), separated from the ventricle by an ependymal cell layer. The recent studies used confocal imaging of whole-mount preparations of the LV wall to examine it in more detail.

Embryonic NSCs require contact with the ventricle to maintain their progenitor status. Although it has been reported that some adult NSCs also make ventricular contact, the extent of this interaction was unclear. Mirzadeh et al. and Shen et al. found that many adult type $B$ cells make ventricular contact through tiny apical processes, and in some cases the cell body of these cells was actually within the ependymal layer. Mirzadeh et al. found these ventricular contacts throughout the ventricle wall and found "hot spots" with high numbers of contacts in regions that produce specific types of neurons. The apical processes of the type B cells were surrounded by ependymal cells, forming a "pinwheel" pattern on the ventricular surface. Furthermore, like embryonic progenitors, the type $B$ cells extended a long basal process that partly associated with migrating neuroblasts before terminating on blood vessels.

These findings imply that contact with blood vessels might also be a key feature of the NSC niche. The three studies revealed a dense plexus of blood vessels in the neurogenic SVZ, and Tavazoie et al. and Shen et al. showed that many dividing cells were intimately associated with these vessels. Migrating neuroblasts were also closely associated with the vasculature.

Shen et al. examined the molecular basis of this association, and showed that cells close to the blood vessels express $\alpha 6 \beta 1$ integrin, a major receptor for laminin, which is expressed by blood vessels. Blocking $\alpha 6 \beta 1$ integrin's binding to laminin prevented NSCs from attaching to endothelial cells in vitro, indicating that this interaction might keep dividing cells close to vessels in vivo.

The close association between NSCs and blood vessels suggests that vascular signals might regulate proliferation. Indeed, Tavazoie et al. showed that the blood-brain barrier was modified in neurogenic SVZ regions: gaps between astrocytic end-feet gave proliferating cells access to the vasculature, and a small-molecular-mass compound perfused into the blood was able to enter the SVZ.

Tavazoie et al. provided more evidence for the role of the vasculature in regulating NSC proliferation: when they ablated NSC progeny they found that most regenerative NSC proliferation occured near blood vessels. Furthermore, Shen et al. showed that blocking $\alpha 6 \beta 1$ integrin's interactions with laminin in vivo increased proliferation in the SVZ. These results suggest that the vasculature can both support and control SVZ proliferation.

Together, these studies provide a detailed picture of the microenvironment of the NSC niche and highlight the importance of the ventricle and vasculature for the regulation of NSC function. It will be interesting to identify the signalling factors that contribute to this regulation.

Katherine Whalley

ORIGINAL RESEARCH PAPERS Mirzadeh, Z. et al. Neural stem cells confer unique pinwheel architecture to the ventricular surface in neurogenic regions of the adult brain. Cell Stem Cell 3, 265-278 (2008) | Tavazoie, M. et al. A specialized vascular niche for adult neural stem cells. Cell Stem Cell 3, 279-288 (2008)| Shen, Q. et al. Adult SVZ stem cells lie in a vascular niche: a quantitative analysis of niche cell-cell interactions. Cell Stem Cell 3, 289-300 (2008)

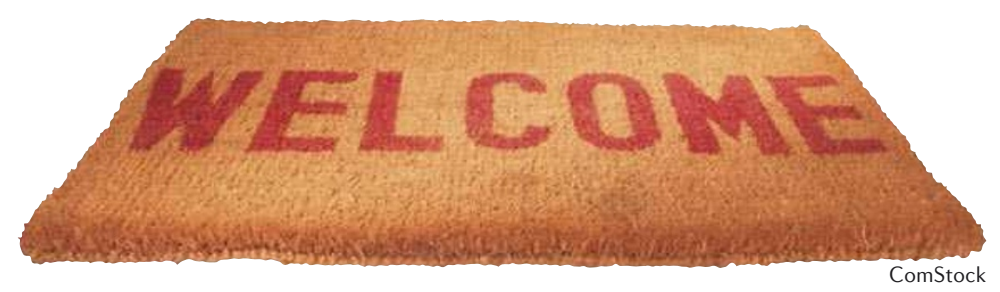

